Verkehrswende und Automobilindustrie Eine schwierige Verbindung

\author{
Die Automobilindustrie ist die Schlüsselindustrie \\ der deutschen Wirtschaft. Nicht nur deswegen \\ kommt ihr im Rahmen der Verkehrswende \\ die zentrale Bedeutung zu. Wie kann die Wende \\ zu einer neuen Kultur der Mobilität gelingen? \\ Von Frank Iwer
}

$I^{\mathrm{n}}$ den letzten Monaten hat die Diskussion über Lage und Zukunft der Automobilindustrie fast schon apokalyptische Züge angenommen. Fast nichts erscheint mehr unmöglich, debattiert wird über Einfahrverbote für sämtliche Dieselfahrzeuge oder auch ein generelles Verbot für Verbrennungsmotoren ab 2030.

\section{Einfahrverbote für Diesel}

Ohne Zweifel: Die Automobilindustrie trägt die Hauptverantwortung für das entstandene gesellschaftliche Klima. Dieselgate - die illegale Manipulation der Software für die Motorsteuerung - war der Katalysator für eine generell kritische Auseinandersetzung mit Zulassungspraktiken oder Differenzen zwischen Testumgebung und realen Fahrsituationen - auch wenn diese formal zulässig waren. Der Vertrauensverlust ist groß und möglicherweise unumkehrbar.

Die IG Metall und die Gesamtbetriebsratsvorsitzende fordern daher schon lange, in einer Transparenzoffensive die realen Verbrauchs- und Emissionswerte zu benennen und zur Grundlage für Verbesserungen $\mathrm{zu}$ machen. Es ist Alltagswissen, dass die Prüfstandwerte in normalen Fahrsituationen nicht erreicht werden können, und eine Binsenweisheit, dass die realen Werte über die Umweltbelastung und Akzeptanz entscheiden, nicht die Testresultate. Aber selbst diese Einsicht erreicht die Automobilunternehmen bestenfalls im Schneckentempo.

\section{Ein Lehrstück für die Zukunft}

Die öffentliche Debatte um die Zukunft des Automobils kreiste in den letzten Monaten fast ausschließlich um den Dieselantrieb. Aber weder der hierzu mit großem Getöse durchgeführte „Diesel-Gipfel“ Anfang August noch das aktuelle Ur- teil des Bundesverwaltungsgerichts hat die wesentliche Frage zufriedenstellend beantwortet: Wie sollen Rechtssicherheit für die Verbraucher/innen, zügige Verbesserungen der Schadstoffbelastung und eine Erreichung der $\mathrm{CO}_{2}$-Zielwerte gleichzeitig realisiert werden, ohne dabei industrie- und beschäftigungspolitische Technologiebrüche zu erzwingen?

Offenbar wird, dass diese Aufgabe einer Quadratur des Kreises gleichkommt, gerade weil es keine schnellen, einfachen und eindeutigen Maßnahmen gibt:

- Hardwarenachrüstungen sind teuer und dauern lange, nicht zuletzt, weil die Kapazitäten für Zulieferungen in Millionen Stück genauso fehlen wie Werkstätten und Prüfstände für die Zulassungen.

- Einfahrverbote, gleich ob mit (Blaue Plakette) oder ohne Kennzeichnung der Fahrzeuge, sind schwierig administrierbar, brauchen umfangreiche Vorarbeiten (zum Beispiel Ausnahmeregelungen), bleiben im Einzelfall rechtlich angreifbar - und sind frühestens 2019 realisierbar.

- Gleichzeitig sinken an allen Messstationen die Belastungen der Stickoxide, Stickstoffoxide und nitrosen Gase $\left(\mathrm{NO}_{\mathrm{x}}\right)$. Auch das Umweltbundesamt erwartet, dass die meisten Städte in ein bis zwei Jahren die Grenzwerte auch ohne Einfahrverbote einhalten können.

- Dennoch wirkt die Diesel-Debatte unmittelbar, die Verkaufszahlen von Dieselfahrzeugen stürzen regelrecht ab, mit spürbar negativen Folgen für die Fahrzeughalter/innen (massiver Wertverlust), die Beschäftigten gerade bei Zulieferer/innen von Dieselkomponenten (starke Unterauslastung, Job-Abbau), aber auch für die Umwelt. Denn die $\mathrm{CO}_{2}$-Belastung ist im letzten Jahr nicht wie geplant um $4 \%$ gesunken, sondern angestiegen.

Diese Widersprüchlichkeiten deuten darauf hin, dass die bisherigen Versuche, immer den jeweils Anderen die Verantwortung zuzuspielen, nicht mehr funktionieren. Eine adäquate Bearbeitung des $\mathrm{NO}_{\mathrm{x}}$-Problems gelingt nicht durch die Suche nach Schuldigen (auch wenn das verständlich sein mag), sondern nur durch ein intelligentes Zusammenspiel aller Akteur/ innen. Wir benötigen eine klare und bundesweite Regulierung durch Einführung einer Blauen Plakette mit definierten technischen Kriterien, welche Fahrzeuge auch künftig in Ballungsräume einfahren dürfen. Wir brauchen erhebliche Anstrengungen der Hersteller/innen, damit möglichst viele Fahrzeuge diese Kriterien auch erfüllen. Wir brauchen in den betroffenen Regionen ausgehandelte und tragfähige Mobilitätskompromisse, die einen bestmöglichen Ausgleich von Interessen der Anwohner/innen und Pendler/innen beinhalten. Das Sys- 
tem von Abgaben, Steuern und Anreizen muss solche Kompromisse unterstützen, ohne soziale Schranken beim Zugang zur Mobilität zu vertiefen.

Es geht um nichts weniger als die Fähigkeit, einen „Systemwechsel unter Vollauslastung“ einzuleiten. Hierzu gehört die Bereitschaft, bestehende Zielkonflikte zu akzeptieren und mit ihnen verantwortlich umzugehen. Denn genau solche Zielkonflikte - zwischen $\mathrm{NO}_{\mathrm{x}}$ - und $\mathrm{CO}_{2}$-Reduzierung, zwischen Anwohner/innen und Pendler/innen, zwischen dem Ausbau des öffentlichen Personennahverkehrs (ÖPNV) und individueller Mobilität, zwischen Verbotsbeschlüssen und Beschäftigungseffekten - werden künftig noch stärker die Debatte prägen und verlangen nach Antworten.

\section{Transformationstreiber der Automobil- industrie}

Die Automobilindustrie steht dabei vor einer dramatischen Transformation, die diese Schlüsselindustrie mit ihren über 900.000 unmittelbar Beschäftigten vielfach durchschütteln wird. Parallele Treiber sind die Megatrends der Digitalisierung und der Dekarbonisierung.

Die Digitalisierung und Automatisierung der Fahrzeuge bewirkt eine viel gravierendere Veränderung als „nur“ die Realisierung neuer Antriebskonzepte. Neue Produkte, neue digitale Dienste, ein Trend der Verbraucher/innen in Richtung „Nutzen“ statt „Besitzen“ von Fahrzeugen sowie das Auftreten neuer Wettbewerber wie Amazon oder Uber sind hier wesentliche Elemente.

Auf der Seite der Produktion sind gleichfalls erhebliche Umbrüche zu beobachten. Neue Automatisierungstrends im Rahmen der Industrie 4.0 halten bereits Einzug in die Fabriken. Und da unter dem Motto eines „globalen Produktionsnetzwerkes“ zugleich die Vor-Ort-Produktion in den großen Weltregionen deutlich zunimmt, verstärkt sich der Druck auf die Beschäftigung im Exportland Deutschland quantitativ wie qualitativ.

Die Klimaschutz-Verpflichtungen aus den Pariser Abkommen sind der zweite zentrale Treiber für die Transformation der Automobilindustrie und damit Beschleuniger für neue Antriebstechnologien, insbesondere für die E-Mobilität. Neue Pkw und leichte Nutzfahrzeuge müssen bis zum Jahr 2020/21 den Grenzwert von 95 Gramm $\mathrm{CO}_{2}$ pro Kilometer im Flottendurchschnitt einhalten, und im Herbst hat die Kommission für den Zeitraum bis 2030 eine Reduzierung um weitere 30\% vorgeschlagen. Deutschland hat im Rahmen des „Klimaschutzplans“ als Zielgröße beschlossen, die $\mathrm{CO}_{2}$-Emission im Verkehrssektor bis 2030 um 40 bis $42 \%$ gegenüber $1990 \mathrm{zu}$ senken.

Solche Zielgrößen verlangen zwingend eine möglichst breit angelegte Veränderung der Antriebstechnologien. Dazu müssen sowohl deutliche Verbesserungen der konventionellen Antriebe auf Basis von Verbrennungsmotoren (die ja auch 2030 noch den Hauptanteil der Neufahrzeuge stellen werden) als auch ein systematisches Hochfahren alternativer Antriebskonzepte angegangen werden.

Der Verbrennungsantrieb bietet nach Einschätzungen der IG Metall noch ein $\mathrm{CO}_{2}$-Minderungspotenzial von 12-15\%; wir setzen uns in den Unternehmen zusammen mit den Betriebsräten dafür ein, diese Potenziale auch real anzugehen und zu heben. Zugleich drängen wir darauf, auch die nötigen Entwicklungen und Investitionen zur Erschließung der neuen Antriebskonzepte bereitzustellen, denn nur dadurch können die ambitionierten politischen Vorgaben erreicht werden. Wir halten es für erforderlich und möglich, dass europaweit bis 2030 circa ein Drittel aller Neufahrzeuge einen solchen alternativen Antrieb aufweist.

\section{Die Industrie alleine kann es nicht richten}

Für die Branche wird das nicht einfach: Seit 125 Jahren haben die deutschen Hersteller, ihr breites Zuliefernetzwerk und die Forschungs- und Hochschullandschaft eine technologische Vorreiterrolle bei den konventionellen Antrieben. Diese war der Schlüssel für die großen Markterfolge gerade in den letzten Jahren und damit auch für die Stabilität im Bereich der Beschäftigung.

Das stellt sich bei den neuen Antriebskonzepten vielfach anders dar. Ob in der Hybridtechnik, bei Elektromotoren oder dem Batterie-Know-how: in allen Schlüsseltechnologien gibt es zum Teil signifikante Vorsprünge bei Hersteller/innen und Zulieferer/innen aus Ostasien. In Deutschland sind die Beschäftigungseffekte dieser Antriebskonzepte daher mittelfristig nicht positiv. Die Realisierung eines 30\%-Anteils von E-Fahrzeugen bis 2030 würde allein in Deutschland einen Abbau von circa 50.000 Arbeitsplätzen nach sich ziehen, ganz zu schweigen von der Notwendigkeit, Tausenden Beschäftigten die nötigen veränderten Qualifikationsinhalte zu vermitteln.

Dennoch setzen wir uns in den Betrieben der Automobilindustrie massiv für diesen Strukturwandel hin zur E-Mobilität ein. Denn ein Scheitern würde letztlich bedeuten, dass diese Leitbranche international immer weiter an Bedeutung verliert - und damit wäre auch eine Sicherung von Beschäftigung pure Illusion.

In Summe bedeutet dies eine große und schwierige Transformation. Von einem erfolgreichen Strukturwandel der Automobilindustrie kann nur dann gesprochen werden, wenn es gelingt, die anspruchsvollen umwelt-, industrie- und beschäftigungspolitischen Ziele im Gleichgewicht zu halten, hierfür tragfähige gesellschaftliche Mehrheiten zu gewinnen und unvermeidliche Zielkonflikte im Interesse der Beschäftigten sachgerecht zu lösen.

\section{Von der Antriebs- zur Mobilitätswende}

Zugleich wird offenkundig, dass die Automobilindustrie allein die nötige Wende der Antriebskonzepte nicht realisieren kann, zu viele Punkte sind ungeklärt: zunächst die öffentlich 
diskutierten Themen Verhalten der Kund/innen, Kaufpreis, Lebenszyklus der E-Fahrzeuge sowie ihre Reichweite. Zum Zweiten offene Fragen rund um Batterietechnologie, insbesondere die Herkunft der Zellen, die indirekte $\mathrm{CO}_{2}$-Belastung bei ihrer Herstellung sowie die Rohstoffproblematik (vor allem Kobalt) und das Recyclingproblem.

Letztlich entscheidend für eine Wende wird jedoch sein, ob und wie die erforderliche Infrastruktur bereitgestellt wird. Das meint nicht nur die Abdeckung mit Ladesäulen, sondern wesentlicher noch die Kapazität und Lastfähigkeit der Stromnetze. Dies verweist auf ein brisantes politisches Problem: Die klimapolitisch erwünschte Verkehrswende kann nur im direkten Zusammenspiel mit der Energiewende gelingen. So hoch der Wirkungsgrad elektrischer Antriebe im Fahrzeug auch ist, die dazu notwendige Energie muss klimaneutral erzeugt, gespeichert, transportiert und ins Fahrzeug gebracht werden. Solange selbst unklar ist, wie der Strom von den Offshore-Windparks stabil in die Industriezentren kommen kann, sind wir hiervon noch weit entfernt. Für ein konsistenteres Zusammenwirken von Energiewende und Verkehrswende sind neue Formen der Beteiligung - etwa von Unternehmen, Umweltverbänden, Gewerkschaften, Landes- und Regionsvertretern sowie der Forschungsseite - dringend geboten. Denn gerade in Ballungsräumen werden regulatorische Vorgaben immer weniger einfach durchgestellt werden können; sie treffen dort auf tiefgehende Zielkonflikte, die nur in regionalen Strukturen auch identifiziert und austariert werden können.

So wird es insgesamt entscheidend sein, dass alle Akteur/ innen an einem Strang ziehen. Natürlich muss zunächst die Automobilindustrie ihren Beitrag leisten: durch entsprechende und bezahlbare Fahrzeuge sowie die Bereitstellung attraktiver Dienstleistungen. Zwingend ist aber das Zusammenspiel mit einer adäquaten Energie- und Ladeinfrastruktur, zusätzlichen Kaufanreizen und letztlich auch einem veränderten Mobilitätsverhalten vor allem in den Ballungsräumen. Diese größeren Zusammenhänge müssen auch in den regulativen Vorgaben aus Brüssel und Berlin mitgedacht werden, anstatt nur eng auf $\mathrm{CO}_{2}$-arme Antriebskonzepte zu zielen. Dabei muss zugleich die ökonomische, industrielle und beschäftigungspolitische Seite beachtet werden.

\section{Die Automobilindustrie vor ihrem größten Umbruch}

Auch wenn dies angesichts von Skandalen und Fehlverhalten seltsam klingt: Die Automobilindustrie ist die Schlüsselindustrie der deutschen (und europäischen) Wirtschaft. Als seit Jahren prosperierende Branche steht sie nicht nur für wachsende Umsätze, sondern gleichermaßen für eine hohe Innovationskraft, für große Investitionsvolumina und für steigende Beschäftigtenzahlen mit guten, tariflich geregelten Arbeitsbedingungen.

Sie steht jetzt vor einem tiefgreifenden Transformationsprozess, der ihr in den nächsten Jahren große Anstrengungen

\section{„Entscheidend für eine \\ Mobilitätswende wird jedoch sein, ob und wie die erforderliche Infrastruktur bereitgestellt wird."}

abverlangen wird. Im Zuge von Digitalisierung und Dekarbonisierung werden sich Produkte und Technologien, Produktionsverfahren, Kooperationspartner/innen und Wettbewerber/ innen, Geschäftsmodelle, Mobilitätskonzepte sowie Formen der nationalen und internationalen Arbeitsteilung verändern. Tradierte Erfolgsfaktoren stehen in den nächsten Jahren vor großen Bewährungsproben und sind keine Gewähr dafür, dass die deutsche Automobilindustrie ihre führende Rolle auch beibehalten kann. Das gilt insbesondere für die vielen Hundert kleineren und oft hoch spezialisierten Unternehmen der zuliefernden Industrie, die häufig nicht über die notwendigen Ressourcen oder die Innovationspotenziale verfügen.

\section{Transformationsprozess als gemeinschaft- liche Aufgabe}

Es ist daher entscheidend, diesen Transformationsprozess Hand in Hand als eine gemeinschaftliche Aufgabe anzugehen. Denn die Folgen im Falle eines Scheiterns wären immens: Zigtausende Arbeitsplätze, der gesamte Industriesektor sowie die Forschungslandschaft und die öffentlichen Finanzen - sie alle wären massiv betroffen. Deshalb kann die Devise nur lauten: Wir dürfen keine Zeit mehr verlieren.

AUTOR + KONTAKT

Frank Iwer leitet den Bereich strategische und politische Planung für den Vorstand der IG Metall. Er ist stellvertretender Vorsitzender des Aufsichtsrats der ZF Friedrichshafen AG und Vorsitzender des Vereins N3tzwerk Zukunft der Industrie.

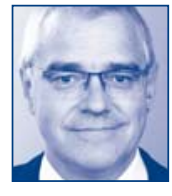

IG Metall Vorstand, Bereich Erster Vorsitzender, Wilhelm-Leuschner-Str. 79, 60329 Frankfurt am Main. Tel.: +4969 6693-2408, E-Mail: maren.rueger@igmetall.de 